\title{
Emergent synchronisation properties of a refrigerator demand side management system
}

\author{
Enrique Kremers* \\ European Institute for Energy Research (Electricité de France 83 Karlsruhe Institute of Technology), Emmy-Noether-Strasse 11, 76131 \\ Karlsruhe, Germany \\ José María Gonzalez de Durana, Oscar Barambones \\ E. U. de Ingeniería de Vitoria-Gasteiz, Universidad del País Vasco, Nieves Cano 12, 01006 Vitoria, Spain
}

\begin{abstract}
In order to analyse the possibilities of improving grid stability on island systems by local demand response mechanisms, a multi-agent simulation model is presented. To support the primary reserve, an under-frequency load shedding (UFLS) using refrigerator loads is modelled. The model represents the system at multiple scales, by recreating each refrigerator individually, and coupling the whole population of refrigerators to a model which simulates the frequency response of the energy system, allowing for cross-scale interactions. Using a simple UFLS strategy, emergent phenomena appear in the simulation. Synchronisation effects among the individual loads were discovered, which can have strong, undesirable impacts on the system such as oscillations of loads and frequency. The phase transition from a stable to an oscillating system is discussed.
\end{abstract}

Keywords: complex systems, electrical grid frequency, emergence, multi agent simulation, spontaneous synchronisation phenomena, under-frequency load shedding.

\section{Introduction}

Interconnected continental power grids such as the European $\mathrm{UCTE}^{1}$ or the North-American power grid offer a large degree of resilience due to their extension and large amount of control mechanisms. The frequency of these grids is usually largely stable and shows only small variations during regular operation. On island systems however, the stability is drastically reduced. Small, selfsufficient electrical systems have been traditionally supplied with high reactive, rather small or medium sized production units. Frequency instabilities are a common issue here. The frequency-response reserve is the first stage of reaction to a frequency drop by providing additional power within the first seconds. The frequency drop is detected when the generator speed slows down, which is compensated by increasing the thermal power immediately. A support of this reserve by demand side management (DSM) could help to improve the stability of the system, by providing additional power for frequency-response.

\footnotetext{
* Corresponding author

Email addresses: kremers@eifer.org (Enrique Kremers ), josemaria.gonzalezdedurana@ehu.es

(José María Gonzalez de Durana), oscar.barambones@ehu.es (Oscar Barambones)

${ }^{1}$ UCTE: Union for Coordination of Transmission of Electricity
}

\subsection{The demand side as a support for frequency stability}

The use of demand side management (DSM) has been widely discussed for different purposes to enhance the performance and avoid critical situations of the power grid $[1,2]$. Hence, DSM allows, among others, to reduce peak loads or perform load shifting. These mechanism can support the integration of fluctuating renewable energy sources such as wind power [3]. Further, DSM can be used to support the primary or frequency-response reserve, which is usually handled by the production side only. The frequency as an instantaneous indicator of the balance between production and demand allows to quickly react to unplanned imbalances of the grid.

In order to support the primary reserve, a highly reactive demand response is needed. As soon as an abnormal frequency drift is detected, the demand side can react by adding or reducing the power consumption. In contrast to classical reserve mechanisms, usually when talking about the demand side we are dealing with a large number of small consumers, rather than with a big dispatchable power plant. In this example we will choose refrigerators, as they are an appliance with a very high penetration rate and, even without having high peak powers, it is usually continuously plugged to the grid. Refrigerators offer the possibility to operate as a thermal storage medium, and this can help to respond in a flexible way to the demand of the energy system. 
Different approaches on DSM concerning refrigerators have been proposed during the last years. Few simulations though can be found. Notably [4] uses a simplified refrigerator model in order to simulate a large number of them, proposing a dynamic operation strategy by varying the thermostat threshold linearly the grid frequency. However, in any of these approaches, the effect of the different demand side management strategies at system level and with a large penetration has been analysed through a detailed model. Only a few high resolution studies and models exist on domestic demand $[5,6]$, and they are not coupled to an energy system model. In the following, a model which aims to represent a large population of frequency controlled refrigerator is presented, which shows unexpected impacts on the system.

\subsection{The energy system as a complex system}

Complexity science is a recent interdisciplinary field of research that has been mainly studied in the social sciences, biology and physics (but not restricted to these areas), that aims to better understand and analyse the processes of both natural and man-made systems composed of many communicating and interacting entities. A remarkable fact in complex systems is the behaviour at the collective level, under some circumstances, suddently exhibits peculiar, time maintained, features that cannot be directly inferred from the behaviour of the individual parts of the system. This phenomenon is known as emergence. The collective behaviour of comunities like as swarms of birds, ant trails or clapping audiences, for example, exibits emergence.

The electrical energy system is today an interconnected, large scale system with dynamic behaviour over time. It is composed of physical networks for electric power transmission and also communication networks for control and data acquisition, at different levels, spread over vast geographical areas. Different participants from these areas, each with their own range of local interests and objectives, all are interacting with the energy system. These factors indicate the energy system can be approached as a complex system.

In the course of this article, it can be seen how through a complex modelling and simulation approach, the emergent phenomenon called spontaneous synchronisation can be detected in an electrical system.

\section{Modelling a refrigerator}

The refrigerator is a device based on a vapour compression cooling system whose main components are the condenser, the evaporator, the compressor and the expansion device. It can be represented by the thermal model described in the following, which can be coupled to other modules (see Figure 1).

In this study we have neglected the transient effect of the thermodynamic Rankine cycle so the refrigerator

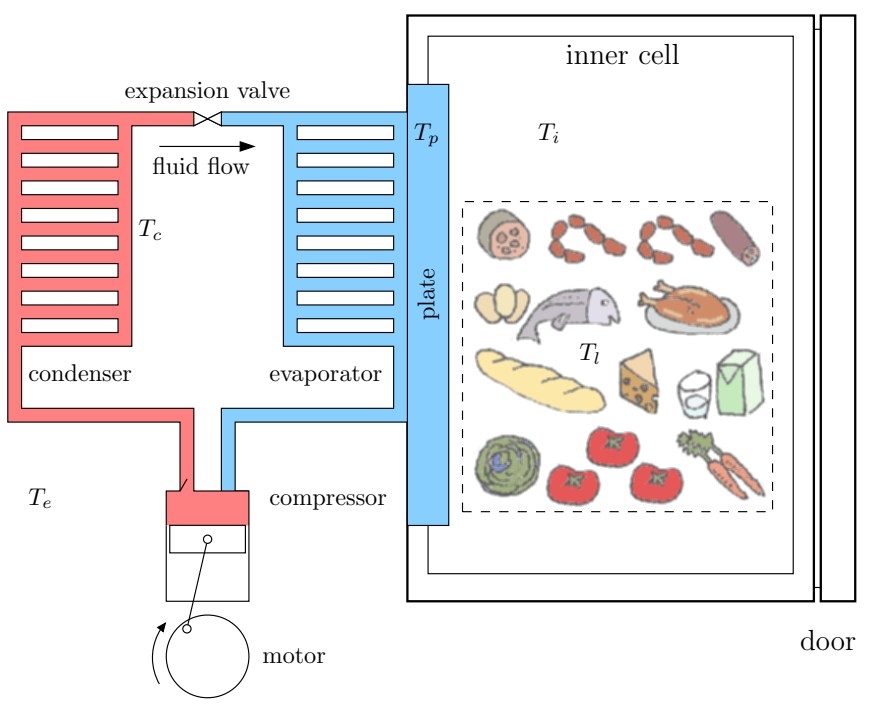

Figure 1: Operating principles of a refrigerator with its components.

model is based only on the fluxes which characterise the heat transfer processes through its internal cell, the evaporator and the exterior, leading to a system of ordinary linear differential equations, as described in [7].

This model was extended with door opening and the effects of the content. Door opening have a large impact on the internal air temperature, especially when the refrigerator is situated in a warm environment. The food and drinks charged in the refrigerator also are relevant for the dynamics and inertia of the inner cell temperature, functioning as a heat storage.

The evolution of the internal temperature is described by adding $T_{\text {door }}^{\prime}$ to the above cited model, to take into account the direct air exchanges due to door openings, giving,

$$
\frac{d T_{i}}{d t}=\frac{T_{e}-T_{i}}{\tau_{e-i}}+\frac{T_{p}-T_{i}}{\tau_{p-i}}+\frac{T_{l}-T_{i}}{\tau_{l-i}}+T_{d o o r}^{\prime}
$$

where $T_{i}[\mathrm{~K}]$ is the internal cell temperature, $T_{e}[\mathrm{~K}]$ the temperature of the environment, $T_{p}[\mathrm{~K}]$ the temperature of the evaporator plate, $\tau_{e-i}, \tau_{p-i}$ and $\tau_{l-i}[\mathrm{~s}]$ are time constants, $T_{l}[\mathrm{~K}]$ is the average temperature of the assumed content in the refrigerator and $T_{d o o r}^{\prime}[\mathrm{K} / \mathrm{s}]$ is the temperature gradient which describes the effect of a door opening.

The variation of the temperature of the evaporator plate is obtained by

$$
\frac{d T_{p}}{d t}=\frac{T_{i}-T_{p}}{\tau_{i-p}}-\frac{\delta_{c} Q_{f}+Q_{e x t}}{C_{p}}
$$

where $\tau_{i-p}[\mathrm{~s}]$ is a time constant and $Q_{f}[\mathrm{~W}]$ is the cooling capacity of the plate, $\delta_{c}$ is a switching control variable with values 0 and 1 , reflecting the thermostat state (Figure 2$), C_{p}[\mathrm{~J} / \mathrm{K}]$ is the thermal capacity of the evaporator fluid and $Q_{\text {ext }}[\mathrm{W}]$ is an exponential function of time which describes the effect of released thermal power after 
the compressor switches off and is explained in detail in [7]. The thermostat is a device that is modeled as a state machine with two states, off and on.

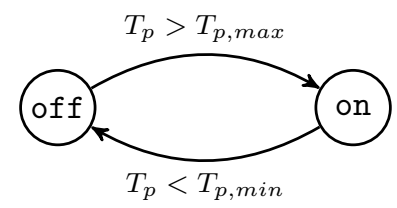

Figure 2: Thermostat state machine

Being in the off state, the system puts the control signal $\delta_{c}=0$ and remains in it until the moment when the temperature $T_{p}$ reaches the value $T_{p, \max }$ which switches to the on state. Being in the on state, the system puts $\delta_{c}=1$ and remains in it until the moment when the temperature $T_{p}$ goes below the minvalue $T_{p, \min }$ which switches to the off state.

The opening of the refrigerator door creates an immediate heat exchange between the environment air temperature and the interior, usually by warmer air flowing into the cell. This heat exchange is added to Equation 1 as an additional term:

$$
T_{\text {door }}^{\prime}=\frac{\delta_{d}\left(T_{e}-T_{i}\right)}{\tau_{d}}
$$

The term is characterised by $\delta_{d}$ which expresses the heat exchange due to door opening. $\delta_{d}=1$ only if the door is open; if it is closed it equals zero. The time constant $\tau_{d}[\mathrm{~s}]$ represents the heat transfer by air exchange between the environment and the inner cell.

In order to recreate the uncertainties of door opening, a stochastic model was included to represent the human behaviour causing door opening. This door opening agent is able to control the refrigerator door and is embedded in the refrigerator. The model is based on a discrete event state-chart, which triggers the $\delta_{d}$ values of the door heat transfer process in Equation 3.

The agent model represent a random opening of the door distributed exponentially over the day, which is characterised by an an average number of door openings per day $n$. The duration of an opening is distributed normally with a mean time $\mu_{\text {doDuration }}$ and the standard deviation $\sigma_{\text {doDuration }}$ of the door opening. The values chosen by default were taken from a survey study [8]. An average of $n=15$ openings per day was taken, with an average duration of $\mu_{\text {doDuration }}=20 \mathrm{~s}$ and a standard deviation of $\sigma_{\text {doDuration }}=50 \mathrm{~s}$.

Further, the effect of the content (food or beverages) in the cell is described by a heat transfer process between the internal cell temperature and the temperature of the content $T_{l}[\mathrm{~K}]$ :

$$
\frac{d T_{l}}{d t}=\frac{T_{i}-T_{l}}{\tau_{i-l}}
$$

where $\tau_{i-l}[\mathrm{~s}]$ is a time constant.
So in summary the refrigerator model consist of a system of the three ordinary lineal differential equations (1), (2) and (4), in the unknown functions $T_{i}(t), T_{p}(t)$ and $T_{l}(t)$, with piecewise continuous control inputs $\delta_{c}(t)$ and $\delta_{d}(t)$, that can be solved for some given initial conditons $T_{i}(0), T_{p}(0)$ and $T_{l}(0)$ using any standard ODE solver.

The model has been implemented in Anylogic, a multi approach simulator that allows combining different modelling methods in the same model. To represent the differential equations, the System Dynamics (SD) AnyLogic paradigm is used. In [9] it is described how SD can be used to model classical electromechanical systems which are continuous models based on ordinary differential equations (ODE). AnyLogic includes a very reliable hybrid solver [10] which is able to combine these continuous systems with discrete models, which create discontinuities in solving of ODEs over time.

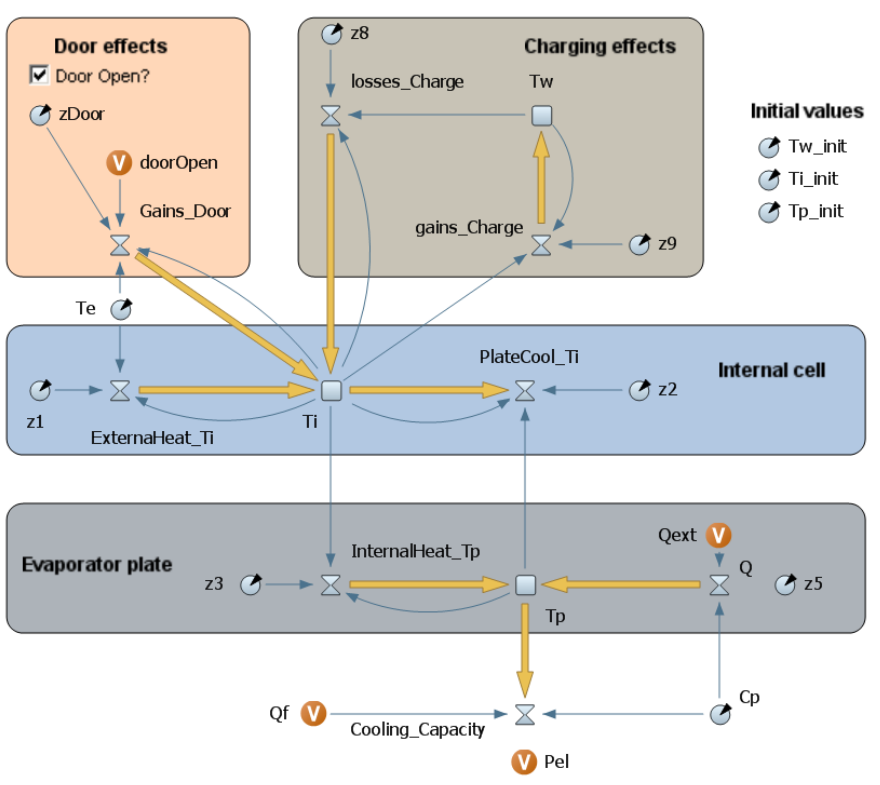

Figure 3: Refrigerator model implemented in System Dynamics.

Figure 3 shows the refrigerator model in the AnyLogic SD graphical user interface, showing two stock objects $T_{i}$ and $T_{p}$ for the unknown functions of their same names and another one $T_{w}$ for the unknown function $T_{l}(t)$. Some additional elements such as variables, flow variables and parameters, necessary to complete the model, are also shown in the figure. The refrigerator parameters were calibrated using the OptQuest optimising engine based on measurements realised on a real refrigerator. This allowed us to verify the validity of the model. An example comparing the measured and simulated values is provided in Figure 4.

Figure 5 shows the simulation results for one single refrigerator, in terms of electrical power and internal temperature. 


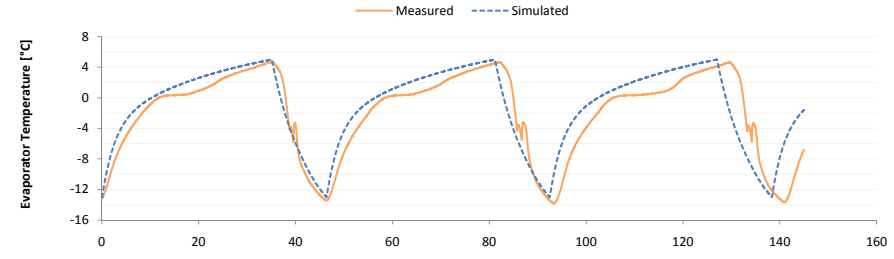

Figure 4: Measured vs. simulated values for the evaporator temperature $T_{p}$. The calibrated model shows a good fit with the observed values, which were measured on a real refrigerator.
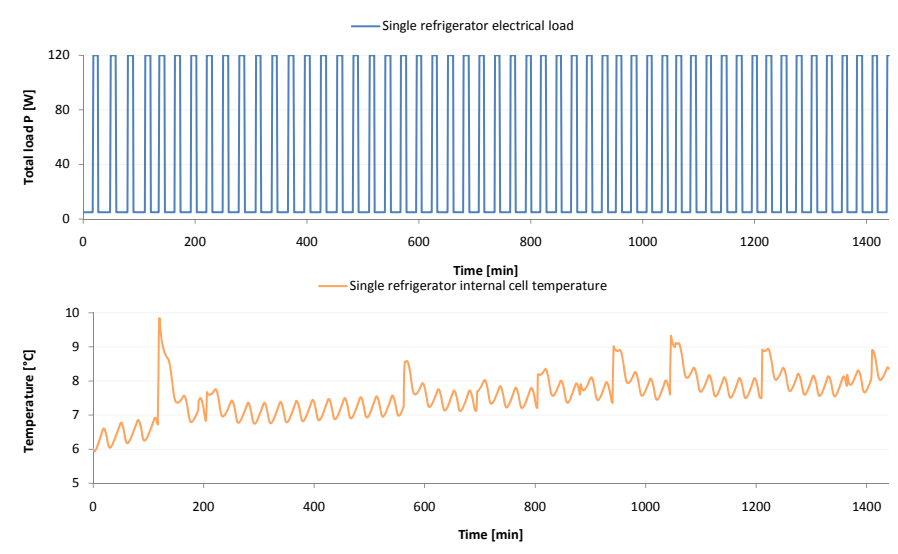

Figure 5: Simulation results for one refrigerator for $24 \mathrm{~h}$. The load curve follows rectangular pulses due to the cyclic operation of the refrigerator. The temperature of the inner cell is maintained at around $7-9^{\circ} \mathrm{C}$. The sharp increases in temperature are due to simulated door opening.

\section{Agent based modelling of the refrigerator pop- ulation}

Agent-based modelling is a technique that recently is gaining more and more importance. An agent-based model combines the use of small, reproducible entities called agents, that interact among themselves and with an environment, leading to complex system behaviour. They are commonly used to obtain decentralised solutions where a central controlled solution method is not applicable. These include open or at least very dynamic environments, systems constituted naturally by agents and systems that have to be easily extendible or scalable [11].

A further advantage of agent-based modelling is that it can be easily combined with other approaches, used to describe the behaviour of the agents. A further advantage of "multiagent modelling" is that it can be easily combined with other approaches used to describe the behaviour of the agents. So, for example, an ordinary differential equation solver, possibly combined with other discrete event algorithms, can be used to model the behaviour over time of each agent.

Considering the fact that the electrical system is composed of many heterogeneous entities, a multi-agent approach seems reasonable. To represent this heterogeneity, the agents can be parametrised in a different way, and so reflect a variety among the population. Each agent is modelled individually, thus no averaged or higher level model is used. This requires on the one hand a greater computational power, but on the other allows high resolution models of the system, where individual values of each entity can be recovered, to be analysed individually or statistically over the whole or parts of the population.

\subsection{Refrigerator agent}

In this case, each agent represents a refrigerator and consist of the three ODE equations (1), (2) and (4), a statechart for the thermostat device for the control input $\delta_{c}$, and the stochastic "social" door open and close generator for the control input $\delta_{d}$. A discrete control mechanism was also added in order to perform frequency based load shedding, as described in the sequel.

The refrigerator agent is be replicated by some factor (48 in the example of Figure 7) to perform the total population and interact with an energy system model, which allows representing the grid frequency.

Coincidence of loads. When individual loads are added, they can coincide in different degrees. If many of the loads have their peak at the same time, this will be reflected on the aggregated load curve. If the peaks are distributed and do not coincide, the load curve is smoother. The coincidence of the loads at a given time $t$ is

$$
c(t)=\frac{P(t)}{P_{n o m}}
$$

where $P$ is the instantaneous load and $P_{\text {nom }}$ the total installed load (maximum possible load if all individual loads would coincide).

\subsection{Description of the energy system model}

The System Frequency Response (SFR) [12] is a simplified model that describes the incremental behaviour of an interconnected electrical system around some nominal point $\left(P_{N}, f_{N}\right)$ of power and frequency values, when dealing with a larges power disturbances. This model omits many details and ignores small time constants, being useful in approximating the electrical system frequency performance and used by different authors $[13,14]$.

The linear Laplace transform model, represented in Figure 6, is composed of two main blocks in a typical feedback loop configuration. The input variable is the "disturbance input" $P_{d}$, i.e. the system power increment over $P_{N}$, and the output variable is $\Delta f$, the system frequency increment over $f_{N}$. All variables in the model are valued per unit. The upper block, with the accelerating power $P_{a}$ as input and $\Delta f$ as output, performs like a big rotating disk with inertia constant $I$ and damping factor $D$ and represents the "spinning reserve" of the generator. The lower feedback block represents the mechanical regulation using $\Delta f$ as input and feeding the mechanical power $P_{m}$ as control action.

Despite of the model simplicity, the comparison with other system disturbances and detailed simulations, as shown 


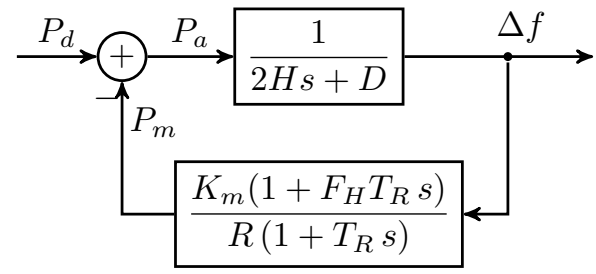

Figure 6: SFR model with Disturbance Input

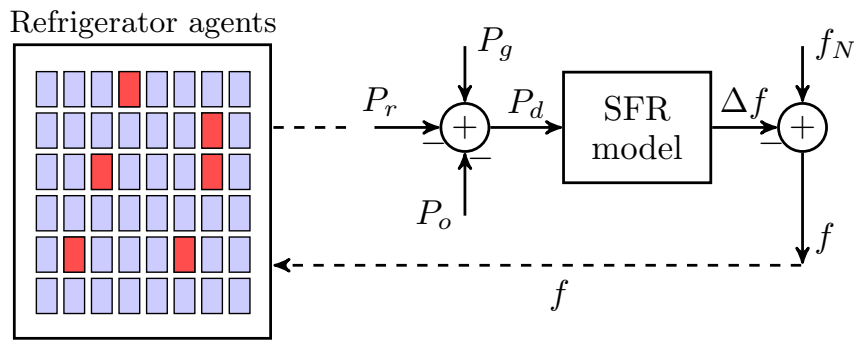

Figure 7: Integral system model: the multi-agent model of the refrigerator population interacts through power and frequency with the simplified energy system model. The aggregated load curve of the refrigerators affects the frequency of the system, which is again used at the refrigerator level in order to perform UFLS. The model represents the system interactions in a dynamic way, as both effects of frequency and power are inherently coupled.

by [12] are rather encouraging. The parameters of the SFR models were set to typical values shown in [12].

Based on the population of refrigerators described above, we create an integral model (Figure 7) coupling the population to the SFR model, which is implemented also in SD. A simplified behaviour of an energy system was considered. The total production of the system was assumed to be constant, as well as the rest of the demand. In fact, the only powers varying in the model are the refrigerators. This simplification allows us to analyse the direct impact of a refrigerator population on the frequency.

The disturbance power is defined by $P_{d}(t)=P_{g}(t)-$ $P_{r}(t)-P_{o}(t)$, where $P_{g}$ is the generated power, and $P_{r}$ is the refrigerator power demand and $P_{o}$ represents all other power demand in the system. A positive disturbance power therefore means an over-generation, and a negative disturbance a over-load of the system.

\subsection{Inclusion of a frequency based load shedding for the refrigerators}

In order to observe the effects of a demand side management mechanism on the refrigerators, a simple and distributed load control was added to each agent in order to perform an UFLS. The operation principle is simple, the refrigerator is unplugged from the grid when a certain frequency threshold $f_{\text {off }}$ is reached, and reconnected when a second threshold $f_{\text {on }}$ is passed over. This aims for disconnecting the load when there is a frequency drop, usually caused by an production failure or a sudden, unforeseen load increase. The refrigerators are disconnected completely from the power source, so the compressor is not able to run any more. As they get reconnected, dependent on the thermostat (the evaporator temperature) will the compressor starts working again or not (see Figure 2).

\section{Simulations and discussion}

In the first simulation, we show the normal operation of the refrigerator population. 250 refrigerators $^{2}$ are simulated with an installed power of $120 \mathrm{~W}$ each. All the parameters of the refrigerators were varied by $5 \%$ in order to recreate a realistic population, in which each refrigerator has a slightly different configuration, as well as different initial conditions (internal temperature, etc.). This creates a heterogeneous population in which each refrigerator has its own state and therefore the individual load curves are particular for each agent. The average total load of the population is $\overline{P_{r}}=11.5 \mathrm{~kW}$ during normal operation, which corresponds to a coincidence of around $c=40 \%$. In order to achieve an impact on a realistic energy system, the population load is scaled up by $s_{f}=1300$, which leads to an average scaled up load of $15 \mathrm{MW}$ (for the refrigerators). The rest of the demanded load of the system was set to $P_{d}=285 \mathrm{MW}$, and the nominal generation power of the system to $P_{g}=300 \mathrm{MW}$. These assumptions correspond to a medium size island system, e.g. as it is the case for the french oversea territory islands.

\subsection{A UFLS scenario}

Production failure is simulated through a sudden drop of $15 \mathrm{MW}$, which get restored linearly in the following $600 \mathrm{~s}$ (see Figure 8). This recreates a failure in a plant with a subsequent reaction of the system (other generators take the dropped load over following the secondary reserve mechanisms). In the first simulation, no UFLS on the refrigerator side was performed. We can observe the aggregated load curve which is rather smooth. The production failure causes the frequency to fall under $49 \mathrm{~Hz}$ for a short time, while the restoration of the power stabilises it within the following 10 minutes.

In the UFLS case the refrigerators disconnect themselves from the system when the frequency falls under $f_{\text {off }}=49 \mathrm{~Hz}$, and we reconnect it as soon it drops above $f_{\text {on }}=50 \mathrm{~Hz}$. As it can be seen in Figure 8, as soon as the frequency falls below the threshold, the complete refrigerator load is released. However, after some time, the load of the refrigerators increases up to twice its average value (around $30 \mathrm{MW}$, which means that almost $80 \%$ of the refrigerators are working at the same time). This rebound effect is due to the hysteresis process which controls the thermostat of the refrigerators. This happens even if the disconnection is only for a few seconds, as this is enough to

\footnotetext{
${ }^{2}$ This number was chosen as a compromise because it still offers reasonable computation times but is high enough to deliver a representative aggregated load curve.
} 

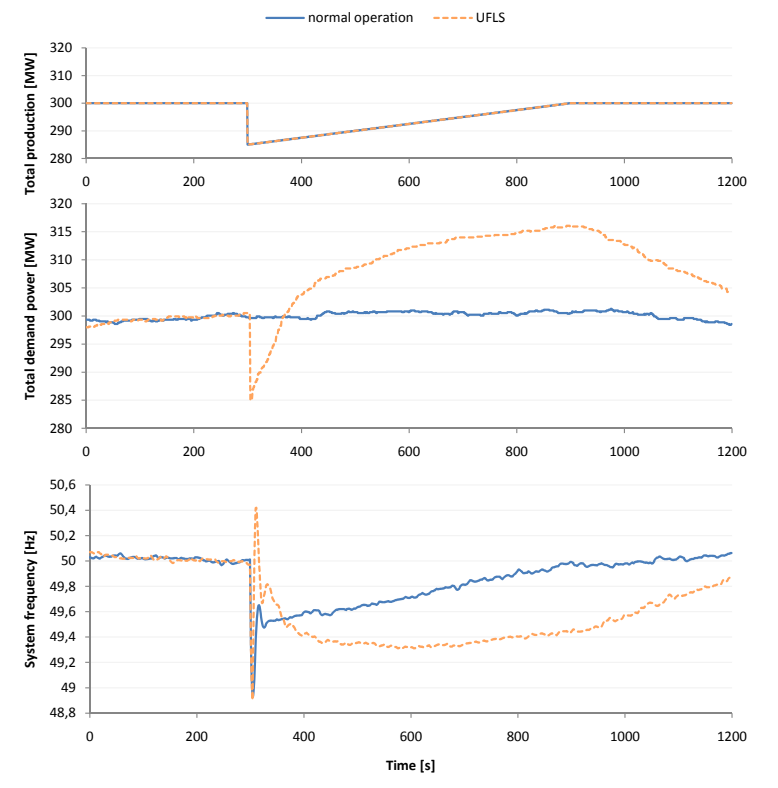

Figure 8: Simulation results for a production failure scenario with and without UFLS.

interrupt the cooling cycle and disconnect the compressor. The refrigerators, which are at the beginning working out of phase, get into a similar phase.

Figure 8 shows the frequency increasing in consequence to the disconnection, and within $6 \mathrm{~s}$ after the disconnection it reaches up to $50.4 \mathrm{~Hz}$. However, due to the increase of the refrigerator demand caused by the rebound (in comparison to normal operation), the frequency recovery is slower than without UFLS.

This very simple UFLS strategy only based on frequency thresholds therefore can help to restore the frequency quickly after an event, but can create a rebound effect due to the coincidence of the loads after the disconnection. This load increase is counter-productive for the frequency stabilisation. Other strategies which do not cause such a rebound, should be taken into account. This can be achieved by reconnecting the refrigerators randomly over a longer time period, for example.

\subsection{Synchronisation and emergent phenomena in the sim- ulations}

A further scenario illustrated in Figure 9 shows the effect of the same load shedding strategy, when increasing the impact of the refrigerator power on the total demand. This was achieved by increasing $s_{f}$ from 1300 to 1760 . As the refrigerators are shed and reconnected a short time after (when frequency drops above $50 \mathrm{~Hz}$ ), they tend to coincide in a higher degree, as describe before (Figure 9). The coincidence has shown to be greater after a load shedding. Yet, no oscillation appears. But as they reconnect again and again, a second under-frequency event takes place, which is not due to a production failure, but to the demand increase of the refrigerators. After some time, the
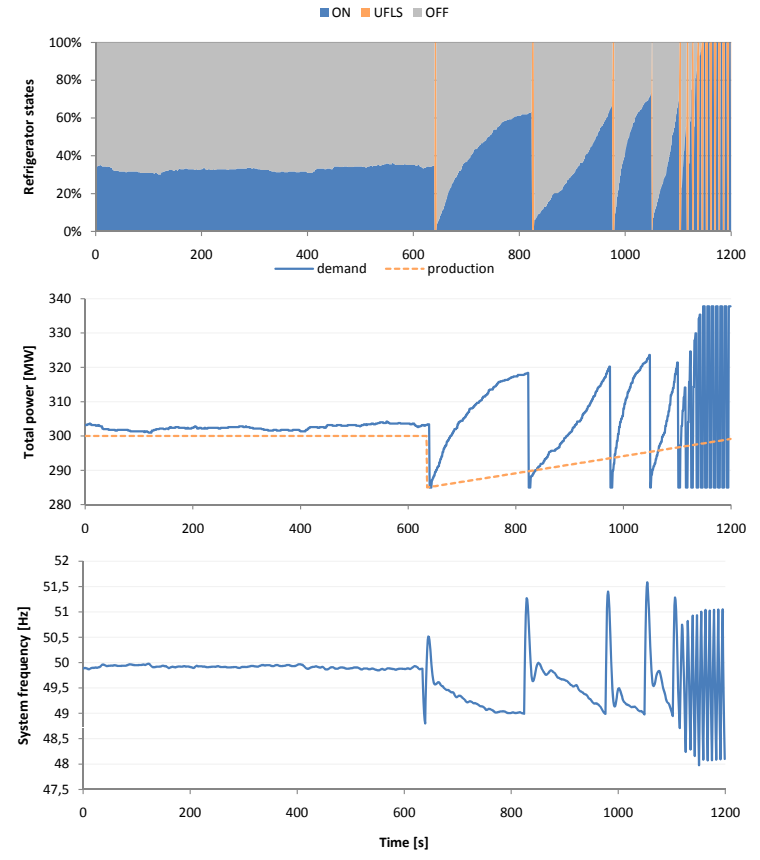

Figure 9: Simulation results for a production failure scenario with UFLS. The system begins to slowly synchronise itself up to a complete oscillation of frequency and loads.

system begins to oscillate with a period of 2-4 $\mathrm{s}$ in the simulated cases. The period seems to be related to the grid frequency response. The origin for the oscillations is the rebound effect explained before. After each reconnection more refrigerators are in phase, which increases the total load and reinforces the subsequent rebound.

The appearance of synchronisation apparently does not depend on the number of individual refrigerators, but rather on the aggregated load that is shed. We could reproduce the effect with relatively small populations of refrigerators (50-300) by scaling up the load. This means that if the total load of the refrigerators which are managed by an UFLS is high enough in relation to the energy systems frequency response, a risk of synchronisation might exist.

The phenomena of an oscillation is not predictable from the individual models of the refrigerators. It emerges in a rather unstable way, which is related to the state of the population (describe by the individual states of each refrigerator), which has a very large degree of freedom. This is typical for complex systems. Due to the used of an agentbased model we were able to recreate a population which is already capable to represent the system as a whole, in a disaggregated way, with its interactions among different levels.

\subsection{Conditions for an emergent oscillation}

Once having identified that the refrigerator system tends to oscillate in some cases, it is interesting to determine when this will occur. Several simulation runs showed that there is no discrete threshold between a stable and an os- 
cillating system, but rather we are faced with a phase transition in which it is more or less probable that the system oscillates.
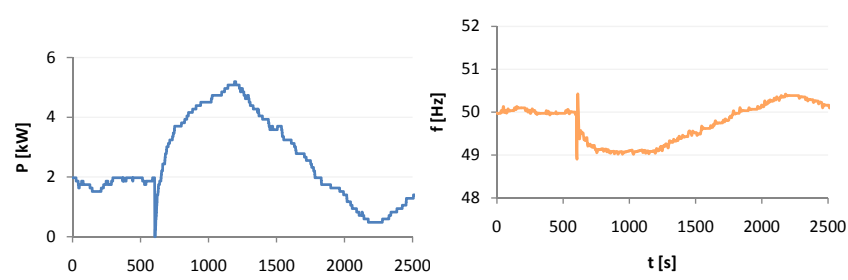

(b) Stable
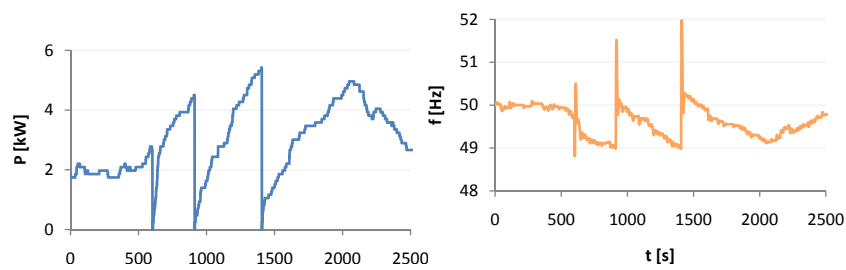

(b) Partial
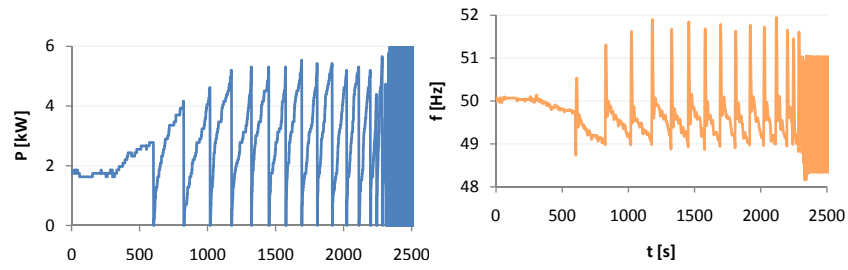

(c) Oscillating

Figure 10: Examples of different regimes of the refrigerator system. On top, the total load curve of the refrigerators and below the corresponding grid frequency. The simulations were performed with the same parameters. Due to the non-deterministic nature of the model and for the given parameterisation, each of the three regimes can appear.

Based on the simulation scenario described so far, a Monte-Carlo evaluation was run to determine the probability of oscillation under different system conditions. First simulation runs suggested that the system oscillates when the total power is high enough to induce a frequency drop that underpasses the UFLS threshold frequency $f_{\text {off }}$. Therefore, for higher scaling factors, oscillation is more likely. The scaling factor $s_{f}$ was varied from 6000 to 12750 in steps of 250 . For each value of $s_{f}, 100$ simulation runs were executed. Three regimes were evaluated:

- Stable: the system responds to the under-frequency event by performing an UFLS only once.

- Partial oscillation: the system responds to the underfrequency event and begins to oscillate more than once, but does not achieve a complete oscillation. After some partial oscillations, the system stabilises again.

- Complete oscillation: the system begins to oscillate and the period becomes shorter up to the moment when it becomes constant.
The probability of each state as a function of $s_{f}$ is shown in 11. As expected, it was found that the probability of staying in a stable system decreases for higher $s_{f}$ values. However, the probability values of partial oscillation are still unstable. It increases up to a maximum of $p($ partial $)=0.11$ and as the phase transition is passed, it decreases again for greater values of $s_{f}$. Even for 150 simulation runs per value, the partial state seems to be uncertain, and the results are not stable. Here lies the edge of chaos, between two phases whose outcome cannot be predicted.

The phase transition forms an s-shaped curve, which is common to other complex systems and appears, for example, in the diffusion processes (Bass model, diffusion of innovations, etc.). This s-shaped curve can be seen in Figure 11.

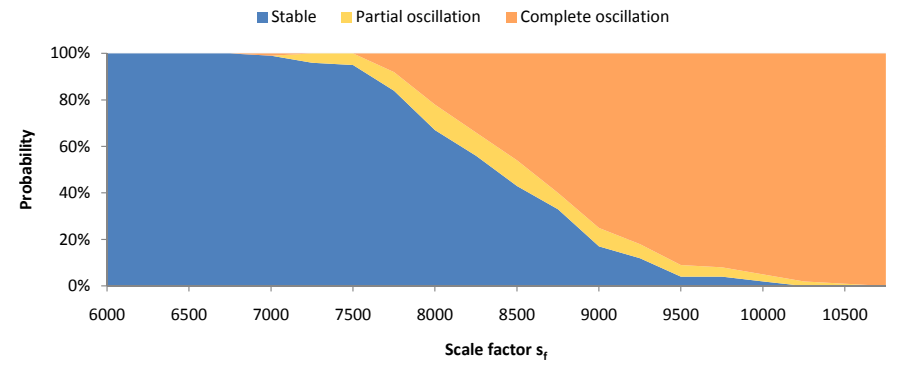

Figure 11: Conditions for a stable, partial and oscillating regime in function of the scale factor. The curve is obtained through a MonteCarlo simulation, in which 100 runs were executed for each value of $s_{f}$.

Knowing more about these phase transitions in real systems can help to further avoid unstable situations and to dimension a system that will stay in a stable state. The risk of a partial transition can be therefore reduced. As performing these kind of experiments on real systems is not possible, simulation can be a powerful tool in exploring behaviour in these conditions. For example, rare events can take place in a regime close to phase transition, but in the real system it would not be possible for sufficient experiments to be performed to attain this level of knowledge and the probability of an oscillation would be very low. However in this case, the risk exists and, through simulation, it can be identified and addressed.

\section{Conclusions}

We represented a population of refrigerators using a multi-agent simulation model and coupled it with a simplified grid model which allows us to recreate the behaviour of the production-demand balance on the frequency. A calibrated and individually validated refrigerator model was taken as a base. A frequency based demand side management unit was added to the refrigerator, which unplugs the refrigerator at under-frequency events marked by a threshold, and reconnects the appliance when the frequency sur- 
passes a second threshold. This represents cross-scales interactions of the system.

A rebound effect was found using a very simple UFLS strategy. Further, synchronisation effects could be captured and analysed. An oscillation of the system if the refrigerator disconnected refrigerator load is high enough could be observed and should be avoided in real devices. A phase transition between a stable regime and an oscillating regime could be observed, in which the system tends to be unstable. In this intermediate state, it is not clear if the system will oscillate or return to stability. The nondeterminism of the implemented model allows to explore these different states of the model. An s-shaped phase transition was found when varying the size of the population and observing the probability of occurence of the different regimes, which is typical for complex systems.

The simulation environment allows to test UFLS strategies virtually in a large scale environment an analyse the impact at a systemic level. For example, in order to avoid dangerous synchronisations, strategies that perform UFLS with variable thresholds (random thresholds, varied over a range) could prevent these type of effects. Individual models or non-interacting models where there is no feedback between the electrical grid and the load management are not able to capture these effects. It has to be noted that this effect only appears with a large number of refrigerators, and thus can only be difficulty analysed in real experimental cases, as the impact on the energy system must be large enough. Further, a laboratory test on a few number of refrigerators would not allow a sufficient impact on the frequency.

\section{Outlook}

Emergence has been studied in other field domains, especially by complexity science, however, few applications of these theories has been made to engineering or energy systems. The properties of the emergent synchronisation should be further analysed. The simulation test suggest that the probability of achieving an emergent oscillation depends on the amount of load (number of refrigerators) that are managed, the properties of the system (frequency response). Further, the model permits to test strategies to avoid these effects, for example by varying the thresholds among the agents, or using a hysteresis process to avoid oscillations.

The period of the oscillation for example, suggests to be a function of the characteristics of the population of the refrigerators, as well as the frequency response. As all refrigerators have different properties, it can't be deduced that there is a common period for all and that this is the reason for the oscillation. Further, the phase transition from a stable regime (no oscillation) towards a completely synchronised regime should be analysed more in detail, as the phase shift has shown to occur sometimes very quickly (some minutes), and others taking much longer (up to an hour).
A relation with the Kuramoto and Nikitin models [15, 16], which are models for the behaviour of a large set of nearly identical coupled oscillators, would be worth to study.

\section{Acknoledgments}

We would like to thank the support of the colleagues of EDF who contributed to the measurements on the refrigerator.

\section{References}

[1] Palensky, P., Dietrich, D.. Demand side management: Demand response, intelligent energy systems, and smart loads. Industrial Informatics, IEEE Transactions on 2011;7(3):381-388.

[2] Albadi, M.H., El-Saadany, E.F.. Demand response in electricity markets: An overview. In: Power Engineering Society General Meeting, 2007. IEEE. 2007, p. 1-5.

[3] Moura, P.S., de Almeida, A.T.. The role of demand-side management in the grid integration of wind power. Applied Energy 2010;87(8):2581-2588.

[4] Short, J.A., Infield, D.G., Freris, L.L.. Stabilization of grid frequency through dynamic demand control. Power Systems, IEEE Transactions on 2007;22(3):1284-1293.

[5] Wright, A., Firth, S.. The nature of domestic electricity-loads and effects of time averaging on statistics and on-site generation calculations. Applied Energy 2007;84(4):389-403.

[6] Widn, J., Wckelgrd, E.. A high-resolution stochastic model of domestic activity patterns and electricity demand. Applied Energy 2009;87(6):1880-1892.

[7] Cerri, G., Palmieri, A., Monticelli, E., Pezzoli, D.. Identification of domestic refrigerator models including cool storage. In: International Congress of Refrigeration 2003. Washington D.C., USA; 2003,.

[8] Laguerre, O., Derens, E., Palagos, B.. Study of domestic refrigerator temperature and analysis of factors affecting temperature: a french survey. International Journal of Refrigeration $2002 ; 25(5): 653-659$.

[9] Viejo Garcia, P., Gonzalez de Durana, J., Barambones, O., Kremers, E.. Quantitative system dynamics: Comparison of modeling techniques for the simulation of electro-mechanical systems. In: International System Dynamics Conference. Washington; 2011,.

[10] Borshchev, A., Kolesov, Y., Senichenkov, Y.. Java engine for uml based hybrid state machines. vol. 2. IEEE; 2002, p. 1888-1894.

[11] Wooldridge, M.J.. An introduction to multiagent systems. Chichester, West Sussex, United Kingdom: Wiley; 2009.

[12] Anderson, P.M., Mirheydar, M.. A low-order system frequency response model. Power Systems, IEEE Transactions on 1990;5(3):720-729.

[13] Lee Hau Aik, D.. A general-order system frequency response model incorporating load shedding: analytic modeling and applications. Power Systems, IEEE Transactions on $2006 ; 21(2): 709-717$.

[14] Kottick, D., Blau, M., Edelstein, D.. Battery energy storage for frequency regulation in an island power system. Energy Conversion, IEEE Transactions on 1993;8(3):455-459.

[15] Kuramoto, Y.. Chemical oscillations, waves, and turbulence. Dover Pubns; 2003.

[16] Nikitin, A., Nda, Z., Vicsek, T.. Collective dynamics of two-mode stochastic oscillators. Physical Review Letters 2001;87(2):024101. PRL. 